\title{
La Influencia de las Expectativas Premigratorias en la Satisfacción Vital en Inmigrantes Latinoamericanas: El Papel del Apoyo Social y la Estabilidad Familiar
}

\author{
Premigratory Expectatives and Vital Satisfaction in Latin-American Inmigrants: The \\ Role of Social Support and Family Stability
}

\author{
Silvia Peñas ${ }^{1}$, David Herrero-Fernández ${ }^{2}$, Laura Merino ${ }^{3}$, Susana Corral ${ }^{4}$ y Ana Martínez- \\ Pampliega 5
}

\begin{abstract}
Resumen
La presente investigación tuvo como objetivo analizar el efecto moderador de la satisfacción marital y el apoyo social en la relación entre las expectativas premigratorias y la satisfacción vital. Para ello se contó con una muestra de 81 mujeres inmigrantes latinoamericanas en España que han reagrupado a su familia. Los resultados muestran que tanto las expectativas premigratorias como el apoyo social y la satisfacción marital correlacionan significativamente con la satisfacción vital. Sin embargo, al analizar el efecto de moderación, sólo la satisfacción marital mostró un efecto significativo, de manera que esta variable amortigua el efecto negativo que tienen las expectativas no realistas sobre la satisfacción vital. Estos resultados pueden tener importantes implicaciones prácticas desde el punto de vista del trabajo psicoterapéutico con personas inmigrantes en el país de destino. Finalmente, se comentan las limitaciones del estudio y se discuten algunas líneas de investigación a futuro.
\end{abstract}

Palabras clave: inmigración, reagrupación familiar, apoyo social, satisfacción marital

\begin{abstract}
The current research aimed to analyze the moderation effect of marital satisfaction and social support in the relationship between premigratory expectative and vital satisfaction. In the study 81 Latin-American immigrant females who had regrouped their families. The results showed that both premigratory expectative and social support correlated with vital satisfaction. However, only marital satisfaction had a significant moderation effect, so this variable muffles the negative effect that non-realistic expectative have on vital satisfaction. These results can have practical implications from the viewpoint of psychotherapeutic work with immigrant people in the incoming country. Finally, limitations of the study are discussed and possible future research lines are proposed.
\end{abstract}

Keywords: inmigration, familiar regrouping, social support, marital satisfaction

\footnotetext{
${ }^{1}$ Doctora en Psicología. Universidad de Deusto, Bilbao, España. Avda. de las Universidades, 24, 48007 Bilbao, España. Tel.. +0034 944139000. Correo: silvia@egintza.com

${ }^{2}$ Doctor en Psicología. Universidad de Deusto, Bilbao, España. Avda. de las Universidades, 24, 48007 Bilbao, España. Tel.: +0034 944139000. Correo: d.herrero1984@gmail.com (autor de correspondencia)

${ }^{3}$ Doctora en Psicología. Universidad de Deusto, Bilbao, España. Avda. de las Universidades, 24, 48007 Bilbao, España. Tel: +0034 944139000. Correo: laura.merino@ deusto.es

${ }^{4}$ Doctora en Psicología. Universidad de Deusto, Bilbao, España. Avda. de las Universidades, 24, 48007 Bilbao, España. Tel: +0034 944139000. Correo: susana.corral@deusto.es

${ }^{5}$ Doctora en Psicología. Universidad de Deusto, Bilbao, España. Avda. de las Universidades, 24, 48007 Bilbao, España. Tel: +0034 944139000. Correo: martinez.pampliega@deusto.es

Revista Iberoamericana de Diagnóstico y Evaluación - e Avaliação Psicológica. RIDEP · No53 · Vol.4 · 91-100 · 2019

ISSN: 1135-3848 print /2183-6051online
} 


\section{Introducción}

La inmigración es un fenómeno sociocultural de creciente importancia en el mundo, que implica las dimensiones de internacionalidad y perdurabilidad en el tiempo (Gregorio, 1998). La movilidad social sigue siendo una de las características de nuestra época. La globalización $\mathrm{y}$, muy especialmente, las transformaciones en los flujos de signos migratorios modifican la naturaleza y el significado de las migraciones contemporáneas. El incremento de las actividades trasnacionales y la configuración de enclaves étnicos (Ariño, 2009) se ven agudizados por las crisis políticas y económicas en los países de origen de las familias migrantes (Ramirez, 1998).

En el caso concreto de España, los datos de inmigración han fluctuado en los últimos 6 años como consecuencia de la situación socioeconómica de cada momento. Según estadísticas oficiales, entre el año 2012 (5,236,030 extranjeros) y el año 2016 (4,417,517 extranjeros) la tendencia era negativa, de forma que cada año el número se reducía con respecto al anterior. Sin embargo, en el año 2017 se incrementó el número de extranjeros, alcanzando la cifra provisional de 4,424,409 personas (INE, 2017). En cualquier caso y según la misma fuente el número de extranjeros en esta franja de años siempre ha supuesto más de un $10 \%$ de la población total española, con lo que constituye un colectivo socialmente relevante.

Dentro del colectivo de inmigrantes, uno de los grupos más importantes es el de inmigrantes latinoamericanos, de la que España es el segundo país que más recibe tras Estados Unidos (Rico, 2006) debido al idioma común y, en cierto modo, a la cultura (Bermúdez, 2004). Dentro de éstos, la mayoría proviene de Ecuador, Colombia, Bolivia y Argentina. En el año 2016 estos cuatro países sumaban 455,692 personas.

Uno de los problemas psicológicos que se han detectado en población inmigrante es la baja satisfacción vital en comparación con personas nativas (Kong \& You, 2013; Moscato, Novara, Hombrados-Mendieta, Romano, \& Lavanco, 2014; Utrera \& Guardiola, 2017). La satisfacción vital hace referencia a un sentimiento emocional básico sobre la evaluación global de la calidad de vida (Diener, Diener, \& Diener, 1995; Diener,
Emmons, Larsen, \& Griffin, 1985; FernándezBerrocal \& Extremera, 2009), que está en función de una serie de atributos psicológicos asociados al buen desarrollo y al ajuste psicosocial de la persona (Basabe, Páez, Aierdi, \& JiménezAristizabal, 2009). Estos atributos a su vez se deben a diversos aspectos, tanto objetivos, por ejemplo el hecho de estar en situación de regularidad administrativa (Moreno-Jiménez \& Hidalgo, 2011), como -principalmente- subjetivos, por ejemplo el ajuste entre las expectativas premigratorias y los logros conseguidos en la nueva sociedad (Neto, 1995; Holgado, Sánchez, \& Navas, 2011). Las expectativas premigratorias constituyen, además, una de las variables más relevantes a la hora de pronosticar el retorno al país de origen o la permanencia en el de destino migratorio, lo que muestra su asociación con la satisfacción vital derivada del proceso migratorio (Bürgelt, Morgan, \& Pernice, 2008). Por tanto, la satisfacción vital en el inmigrante puede pronosticarse, hasta cierto punto, antes de que se produzca la migración, a través del análisis de las expectativas premigratorias. De esta forma, se ha observado que las personas que parten de sus sociedades de origen con bajas expectativas sobre lo que vivirán en el país de destino se sienten más satisfechas y felices incluso en condiciones menos favorables o con menos logros alcanzados en la sociedad de destino que aquéllas que partían de unas expectativas excesivamente altas (Gómez, Villegas, Barrera, \& Cruz, 2007). Finalmente, Las mujeres podrían ser más proclives a experimentar bajos niveles de satisfacción vital en el proceso migratorio, lo cual se ha vinculado a la menor implicación con respecto a los hombres en la toma de decisiones acerca de llevar a cabo el propio proceso migratorio (Hiller \& McCaig, 2007; Rubin, 2013).

Sin embargo, teniendo en cuenta la diferencia temporal entre el momento de generarse las expectativas y el momento de reportar el grado de satisfacción vital en el país de destino, pueden existir variables que moderen esta relación, bien amortiguando el efecto negativo de las altas expectativas sobre la satisfacción vital, bien potenciando el efecto positivo de las expectativas bajas. En este sentido, dos de los principales factores de protección que se han propuesto para mejorar el estado de bienestar general son el 
apoyo social en el país de destino y la satisfacción o calidad marital.

El apoyo social se define como el conjunto de provisiones expresivas o instrumentales (percibidas o reales) que proporciona la comunidad, las redes sociales y las personas del entorno más cercano (Lin, Dean, \& Ensel, 1986), y se ha relacionado con la satisfacción vital en numerosos estudios, tanto de forma genérica (Bai, Yang, \& Knapp, 2018; Guo, 2014) como en el caso concreto de personas inmigrantes (Calvo, Carr, \& Matz-Costa, 2017; Khawaja, Yang, \& Cockshaw, 2016). De esta manera, la red de apoyo constituye uno de los principales recursos con los que cuenta la persona para afrontar cambios y situaciones nuevas, ejerciendo un efecto protector ante sucesos vitales estresantes (Berkman, 1995; Cohen, Underwood, \& Gottlieb, 2000) y facilitando la adaptación al nuevo entorno (Im, 2010). De la misma manera, se ha constatado una mayor probabilidad de presentar preocupaciones excesivas y una mayor vulnerabilidad cuando es escasa la red de apoyo social de la persona migrante (Hepworth \& Larse, 1986; Taylor \& Repetti, 1997).

De forma similar, la satisfacción o calidad marital (Cabrera, Guevara, \& Barrera, 2006) se ha vinculado estrechamente con la satisfacción vital (Bulgan \& Çiftçi, 2017; Carr, Freedman, Cornman, \& Schwarz, 2014; Maglio, Molina, Raimundi, González, \& Schmidt, 2014). La satisfacción marital se define como un estado general de felicidad y satisfacción tanto en el marido como en la mujer en relación a su matrimonio (Thomas, 1997). Por tanto, se trata de la satisfacción global percibida del subsistema marital en relación al compañero/a basado en el nivel de acuerdo que existe entre ambos (Kerig, Cowan \& Cowan 1993; Kurdek, 1996). En las mujeres migrantes, el hecho de estar casadas no constituye un factor de protección en sí mismo. Algunos estudios con población general han mostrado de hecho que en general las mujeres casadas muestran niveles mayores de depresión que las solteras, debido al excesivo estrés relacionado con tener que llevar a cabo múltiples tareas domésticas y laborales (Noble, 2005; Nolen-Hoeksema, 2001). Sin embargo, al analizar específicamente la satisfacción marital sí se observan una relación negativa entre esta variable y el nivel de depresión, incluso en estudios específicos con mujeres inmigrantes (Ahn \& Cho, 2011; Im, 2010).

Por tanto, el objetivo del presente estudio es analizar el efecto moderador del apoyo social y la satisfacción marital en la relación entre las expectativas premigratorias y la satisfacción vital en una muestra de mujeres inmigrantes latinoamericanas. Se espera que niveles elevados de ambas variables moderadoras amortigüen el efecto negativo de las expectativas no realistas y que potencien el efecto de las expectativas realistas sobre la satisfacción vital.

\section{Método}

\section{Participantes}

Para la realización del estudio se contó con la participación de 81 madres latinoamericanas reagrupantes de descendientes residentes en Bizkaia (Comunidad Autónoma del País Vasco, norte de España). Todas las mujeres del estudio han llevado a cabo la reagrupación familiar cumpliendo los requisitos legales exigidos. Las características sociodemográficas de la muestra se detallan en la Tabla 1.

\section{Instrumentos}

Expectativas Premigratorias. Las expectativas premigratorias se midieron de forma retrospectiva a través de un único ítem dicotómico $(1=$ No realistas; $2=$ Realistas).

Índice de Satisfacción Marital (IMS). El IMS (Hudson, 1992) consta de 25 ítems agrupados en un solo factor, y fue desarrollado con el fin medir el grado, la severidad o la magnitud de un problema que un esposo o su pareja tiene en la relación matrimonial. Los ítems hacen referencia a comportamientos, tanto positivos como negativos, de la pareja de la persona que responde sobre ésta (por ejemplo, "mi cónyuge se preocupa por mí"; "mi pareja no me entiende"), y a aspectos propios de la interacción entre ambos miembros (por ejemplo, "mi cónyuge y yo nos llevamos muy bien"), y se responden según una escala tipo Likert de 3 puntos en referencia a la frecuencia de los mismos (1=No, 2=A veces, 3=Sí). En este estudio se aplicó la adaptación española del 
Tabla 1. Características descriptivas de las variables premigratorias estudiadas

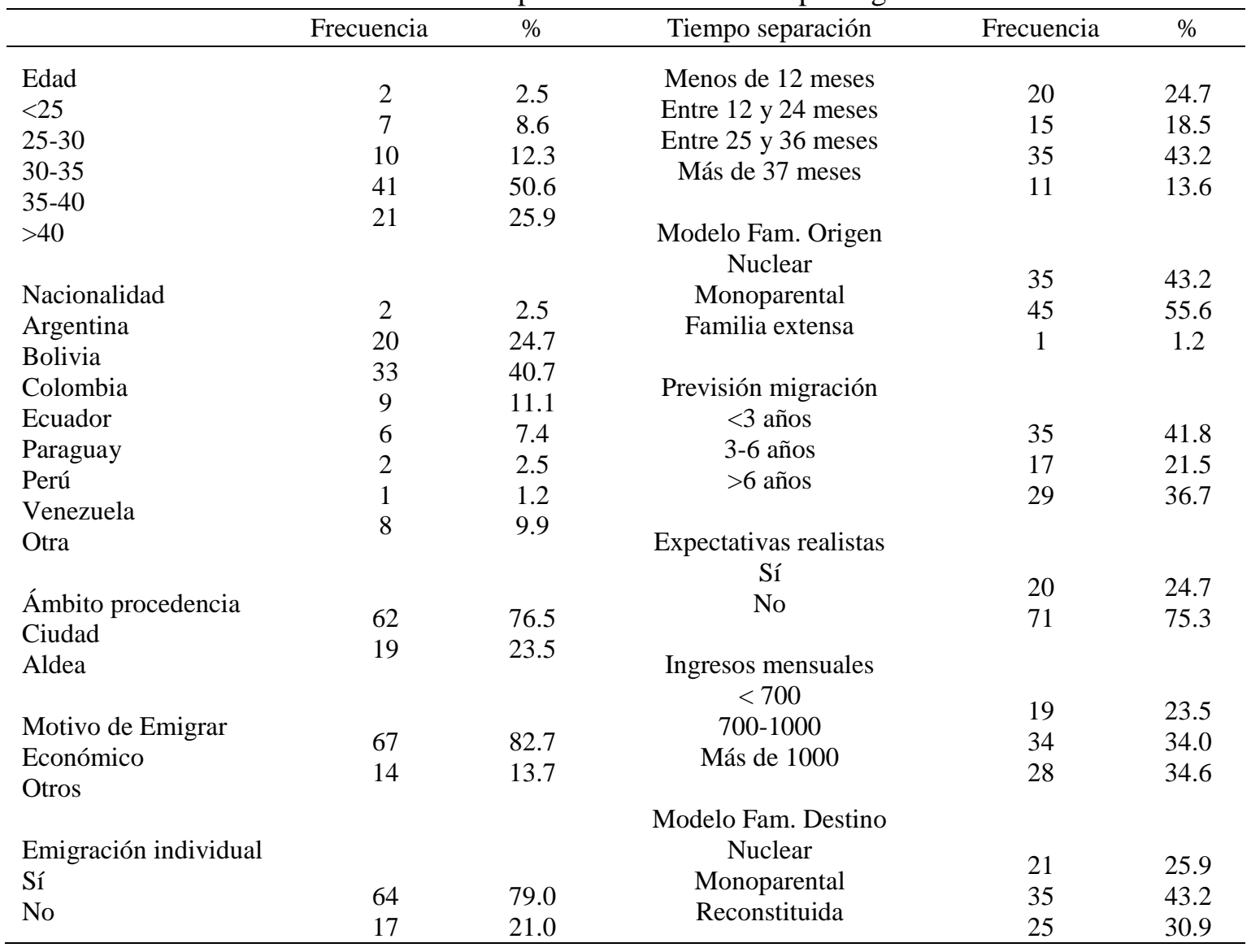

cuestionario (Iraurgi, Sanz, \& MartínezPampliega, 2004), que mostró unas propiedades psicométricas adecuadas. La consistencia interna del instrumento con los datos del presente estudio fue elevada $(\alpha=.95)$.

Cuestionario de Apoyo Social recibido y percibido. El cuestionario de apoyo social recibido y percibido (García \& Hombrados, 2003) consta de 24 ítems que hacen referencia al apoyo recibido por parte de familia, amigos y otros que se contestan según una escala tipo Likert de 1 (rara vez) a 5 (siempre que lo necesito). Este cuestionario es una adaptación realizada sobre el Inventario de Recursos sociales (Diaz-Veiga, 1985). Tiene en cuenta los aspectos relativos a la frecuencia de contacto con la red social, el grado de satisfacción con la relación y el tipo de apoyo (de la familia, de los amigos o de otras personas) que le proporciona la red. La consistencia interna de la escala completa es elevada con los datos del presente estudio $(\alpha=.91)$. Este instrumento se ha utilizado en estudios recientes con población migrante valorando el apoyo social recibido (Domínguez, 2006; Moscato, 2012).
Escala de satisfacción con la vida (SWLS). Esta escala (Diener, et al., 1985) está formada por 5 ítems distribuidos en un único factor con formato de respuesta tipo likert, con 7 alternativas de respuesta ( $1=$ Completamente en desacuerdo; $7=$ Completamente de acuerdo), los cuales evalúan la satisfacción con la vida, entendida como el componente cognitivo del bienestar subjetivo. En este estudio se ha utilizado la adaptación al español (Atienza, Balaguer, Pons, \& GarcíaMérita, 2000). La validez de constructo de la SWLS ha sido analizada recientemente en grandes muestras, habiéndose obtenido unas propiedades psicométricas adecuadas (Arrindell, Heesink, \& Feij, 1999; Díaz \& Sánchez, 2001; Moscato, 2012; Shevlin, Brunsden, \& Miles, 1998). En el presente estudio la consistencia interna obtenida ha sido elevada $(\alpha=.85)$.

\section{Procedimiento}

Para la obtención de un grupo de participantes lo más heterogéneo posible se contactó con diferentes recursos sociales y comunitarios (instituciones, ONG y otras entidades de trabajo con personas inmigrantes). Se divulgó la 
realización de esta investigación a través de todas las instituciones que trabajan directamente con esta población inmigrante en Bizkaia. Cabe señalar que se eligió esta región para llevar a cabo el estudio por ser la región de la comunidad autónoma del País Vasco que mayor número de inmigrantes presenta, concretamente el $48.72 \%$ del total de la comunidad autónoma, con un total de 100,640 personas (Observatorio Vasco de Inmigración, 2018). Por otro lado, se llevó a cabo una difusión radiofónica del estudio en Radio Candela, una de las emisoras de máxima audiencia para el colectivo latinoamericano, y se colocaron carteles informativos de la investigación en locutorios. También nos acercamos a espacios de encuentros habituales de ocio de estas mujeres. Otra de las maneras de captación de mujeres participantes fue a través de la técnica de bola de nieve o cadena, que consiste en contactar con las personas, a partir de otros participantes conocidos, de modo que se va tejiendo una red comunicativa y de participación. Para esta técnica se contó con la colaboración de personas referentes en los diferentes colectivos y recursos de población latinoamericana.

En la divulgación se explicitaban los datos para contactar con el personal investigador tales como el número de teléfono y el correo electrónico. Cuando una madre reagrupante interesada en participar contactaba con el equipo, se fijaba una cita para ofrecerle una explicación más detallada del estudio y otra para la posterior cumplimentación de los instrumentos. Con este objetivo, se realizaron ocho encuentros grupales.

En el contacto directo con las madres participantes se realizaba una presentación de la persona investigadora y una explicación de los objetivos del estudio. A continuación se solicitaba la participación voluntaria y se garantizaba el anonimato, la confidencialidad y la privacidad.

\section{Diseño Metodológico y Análisis de Datos}

El presente estudio se fundamentó en un diseño transversal de tipo correlacional. El análisis de datos se compone de dos partes. En la primera se calcularon los coeficientes de correlación ( $r$ de Pearson) entre las variables medidas. En base a los resultados obtenidos, en una segunda parte se llevó a cabo un modelo de moderación, analizándose simultáneamente el efecto moderador del apoyo social y la estabilidad de pareja en la relación entre las expectativas realistas o no previas al viaje migratorio y la satisfacción vital. Para este análisis se empleó la macro Process 2.16 para SPSS (Hayes, 2013). Como paso previo a la realización de los análisis de moderación se procedió a estandarizar las puntuaciones de las variables independiente y moderadoras, convirtiéndolas a puntuaciones $\mathrm{Z}$, a fin de facilitar el posterior análisis de los efectos condicionales. En el análisis de moderación se considera la existencia de un efecto significativo cuando la interacción entre la variable independiente y la moderadora se asocia de forma significativa con la dependiente. Finalmente, el análisis de moderación se complementó con un análisis del efecto condicional a fin de comprobar el tipo de influencia de las variables moderadoras. Para ello se analizó el tipo de relación entre la variable independiente y la dependiente condicionalmente para diferentes valores de las variables moderadoras, concretamente el valor medio de la distribución, una desviación típica por encima de la media y una desviación típica por debajo de la media (Frazier, Tix, \& Barron, 2004).

\section{Resultados}

Primeramente se calcularon los coeficientes de correlación entre todas las variables. Los resultados mostraron que la satisfacción vital correlacionó positiva y significativamente con las expectativas premigratorias, $r=.23, p=.042$, de manera que aquellas personas que se habían formado unas expectativas realistas mostraban mayores niveles de satisfacción vital. Igualmente la satisfacción vital se relacionó con el apoyo social, $r=.33, p=.003, \mathrm{y}$ con la satisfacción marital, $r=.36, p=.001$. Las expectativas realistas no se asociaron significativamente ni con el apoyo social, $r=.11, p=.320$, y de forma marginalmente significativa con la satisfacción marital, $r=.20$, $p=.071$. Finalmente, el apoyo social y la satisfacción marital no estuvieron significativamente asociados entre sí, $r=.08, p=.494$. El hecho de que la variable independiente no esté relacionada con las moderadoras, ni éstas entre sí, es positivo desde el punto de vista de evitar el efecto de la multicolinealidad (Field, 2005). 
De esta forma, habiéndose comprobado la relación significativa de la variable dependiente (satisfacción vital) con la independiente (expectativas premigratorias) y con las moderadoras (apoyo social y satisfacción marital), se procedió a realizar el análisis de moderación. Los resultados se detallan en la Tabla 2. Tal como se puede observar, el término de interacción creado entre la variable expectativas premigratorias y la satisfacción marital estuvo significativamente relacionado con la satisfacción vital, mostrando un efecto significativo de moderación. Por su parte, el apoyo social no mostró relación significativa con la satisfacción vital, ni al analizarlo individualmente ni en la interacción con las expectativas, de manera que no modera la relación entre expectativas y satisfacción vital.

Tabla 2. Análisis del efecto moderador del apoyo social y la estabilidad de pareja en la relación entre expectativas pre-migratorias y satisfacción vital

\begin{tabular}{lccc}
\hline & $\mathrm{B}$ & E.T.B. & $\Delta \mathrm{R}^{2}$ \\
\hline Paso 1 & & & $.28^{* * *}$ \\
Expectativas & 2.72 & 1.70 & \\
Premigratorias & .43 & 2.13 & \\
$\begin{array}{l}\text { Apoyo social } \\
\text { Satisfacción marital }\end{array}$ & $6.17 * *$ & 2.17 & \\
Paso 2 (interacciones) & & & .04 \\
Expectativas x Apoyo & 1.29 & 1.54 & .01 \\
Expectativas x & $-3.12 *$ & 1.59 & $.04 *$ \\
Satisfacción marital & & & \\
\hline Nota. $\mathrm{R}^{2}=.28, p<.001$. & & \\
$* p<.05, * * p<.01, * * * p<.001$. & & \\
& & &
\end{tabular}

Finalmente, el análisis del efecto condicional de la variable independiente sobre la dependiente en función de distintos valores tomados por las variables moderadoras mostró un efecto significativo cuando la variable satisfacción marital era de 1 desviación típica por debajo de la media y la variable apoyo social valor medio $(\mathrm{B}=5.84, \mathrm{ETB}=2.56, p=.025)$, y cuando la variable satisfacción marital era de 1 desviación típica por debajo de la media y la variable apoyo social 1 desviación típica por encima de la media $(\mathrm{B}=7.13$, $\mathrm{ETB}=2.98, p=.019)$. Además, se observó un efecto marginalmente significativo cuando la satisfacción marital adquiría un valor promedio y el apoyo social un valor de 1 desviación típica por encima de la media $(\mathrm{B}=4.01, \mathrm{ETB}=2.20, p=.072)$. Para mayor claridad de los resultados, el efecto moderador de la satisfacción marital y del apoyo social se representa gráficamente en la Figura 1. En términos generales, una alta satisfacción marital amortigua el efecto negativo que tienen las expectativas no realistas con respecto a la inmigración sobre la satisfacción vital general, anulándose las diferencias entre personas con expectativas realistas y no realistas. Por el contrario, una baja satisfacción marital potencia el efecto negativo de expectativas no realistas sobre la satisfacción vital.

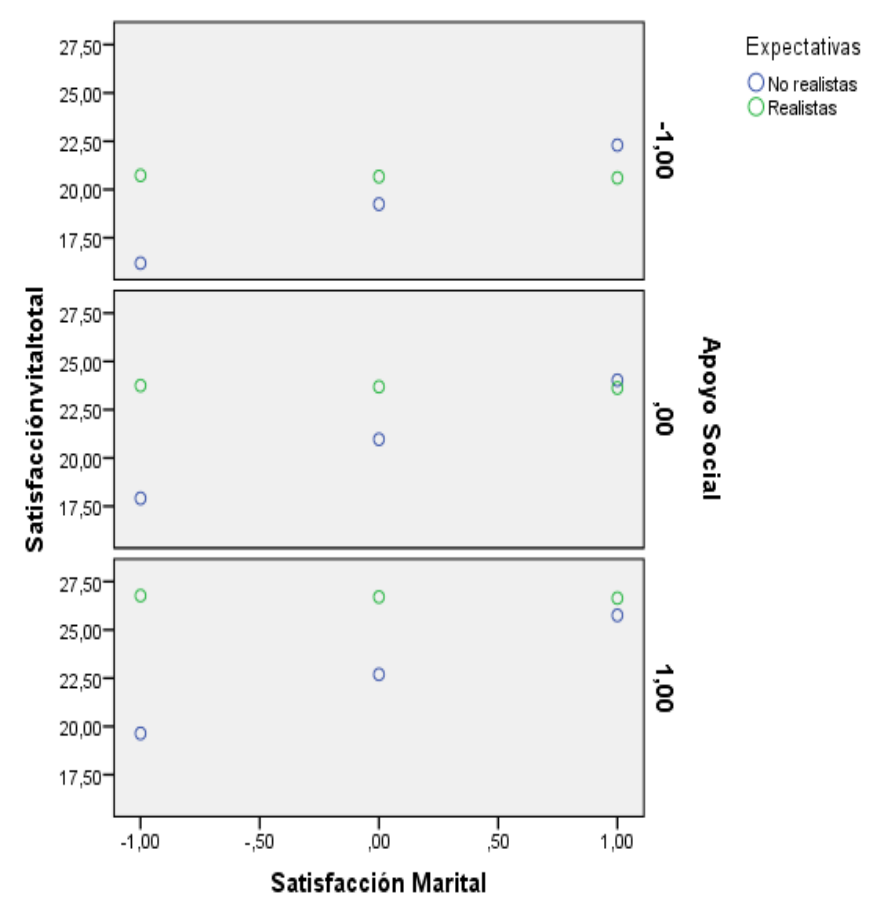

Figura 1. Análisis de efectos condicionales de la moderación de la satisfacción marital y el apoyo social en la relación entre expectativas premigratorias y satisfacción vital

\section{Discusión}

Los procesos migratorios constituyen en muchos casos un factor de riesgo para la salud emocional del inmigrante, debido a la ruptura con el país de origen y con su entorno social y la inculturación en un nuevo contexto social. Si bien en algunos casos hay menos riesgos, como es el caso de los inmigrantes latinoamericanos en España debido a la similitud del idioma y de otros aspectos culturales, tal como se ha expuesto hay variables que implican una menor satisfacción vital general en este colectivo. El objetivo del presente estudio fue analizar el efecto moderador 
del apoyo social y la satisfacción marital en la relación entre las expectativas premigratorias y la satisfacción vital.

Tal como se esperaba, los resultados han mostrado que las personas con expectativas realistas tienden a mostrar niveles más elevados de satisfacción vital que aquellas con expectativas excesivamente altas, lo cual coincide con otras investigaciones similares (Gómez, et al., 2007; Neto, 1995). De la misma manera, al igual que en otros estudios el apoyo social (Calvo, et al., 2017; Khawaja, et al., 2016) y la satisfacción marital (Bulgan \& Çiftçi, 2017; Carr, et al., 2014) se asociaron significativamente con la satisfacción vital. Al analizar el efecto moderador de la satisfacción marital y del apoyo social en la relación entre las expectativas premigratorias y la satisfacción vital, se observó que sólo la primera de las dos variables ejercía un efecto significativo. De esta manera, una satisfacción marital elevada anula las diferencias previamente encontradas en satisfacción marital entre las personas con expectativas realistas y las personas con expectativas no realistas. Estos resultados coinciden con otros estudios que han hallado que la satisfacción marital constituye un factor de protección no sólo de cara a la satisfacción vital, sino también en relación a otras variables más específicas como la depresión (Nho, Kim, Shin, \& Heo, 2017), la ansiedad (Bastida-Gonzalez, Valdez-Medina, Valor-Segura, Fuentes, \& RiveraAragon, 2017; Bayrami, Heshmati, \& Karami, 2011), o el autoconcepto (Cooper, Chassin, \& Zeiss, 1985). En cualquier caso, en el presente estudio se ha analizado como variable de resultado la satisfacción vital, que constituye una variable genérica de calidad de vida (Diener, et al., 1995; Diener, et al., 1985; Fernández-Berrocal \& Extremera, 2009). En futuras investigaciones se debería analizar el papel específico de la satisfacción marital sobre variables más específicas de salud emocional y sintomatología.

Estos resultados pueden tener importantes implicaciones prácticas. Desde el punto de vista de la intervención terapéutica en los países de destino, una vez que el inmigrante ya está asentado en el país de destino, ésta debería orientarse no sólo a trabajar aspectos individuales, sino sobre todo aspectos familiares, de forma que se refuercen las relaciones positivas maritales y esto pueda actuar como factor de protección especialmente en los casos en que el inmigrante venga desde el país de origen con unas expectativas poco realistas sobre lo que será su vida en el país de destino.

Finalmente, este estudio cuenta con algunas limitaciones. En primer lugar, a pesar de la complejidad de la muestra recogida, estadísticamente, el tamaño muestral es relativamente pequeño lo que podría haber interferido negativamente en los resultados. Por tanto, se recomienda replicar los resultados en muestras no sólo más grandes, sino también culturalmente diversas, es decir, de otros países no latinoamericanos. En segundo lugar, la medida de expectativas pre migratorias ha sido dicotómica, lo cual puede restar precisión a los resultados obtenidos al haberla sobre simplificado. Sin embargo, el hecho de no existir instrumentos estandarizadas en castellano para medir esta variable hizo imposible la utilización de una medida que hubiese ofrecido una medición continua de la variable. Además, esta forma de medir las expectativas premigratorias ha sido ya empleada en estudios previos por otros autores (Elgorriaga, Martínez-Taboada, \& Arnoso, 2014). Otros estudios han optado por un enfoque cualitativo para estudiar esta variable (Bürgelt, et al., 2008); sin embargo, de cara a investigaciones futuras se aconseja elaborar instrumentos de medida fiables y válidos para esta dimensión.

En conclusión, el presente estudio ha arrojado luz sobre la importancia de reforzar las relaciones maritales en personas inmigrantes, con el objetivo de mejorar su satisfacción vital. Sin embargo, los resultados han de ser confirmados en futuros estudios que repliquen lo obtenido en muestras más grandes y culturalmente diversas.

\section{Referencias}

Ahn, J. H., \& Cho, J. H. (2011). Psychosocial and cultural predictors of acculturative stress among marriage immigrant women in the Chungcheongnamdo area of Korea. Korean Journal of Family Social Work, 32, 137-176.

Ariño, A. (2009). Estilos de aculturación y encrucijadas de la diversidad cultural. Papers, 94, 115-137. doi:10.5565/rev/papers/v94n0.691 
Arrindell, W. A., Heesink, J., \& Feij, J. A. (1999). The Satisfaction With Life Scale (SWLS): Appraisal with 1700 healthy young adults in the Netherlands. Personality and Individual Differences, 26, 815-826. doi:10.1016/s0191-8869(98)00180-9

Atienza, F. L., Balaguer, D. I., Pons, D., \& García-Mérita, M. (2000). Propiedades psicométricas de la Escala de Satisfacción con la Vida en adolescentes. Psicothema, 12(2), 314-319.

Bai, X., Yang, S. Y., \& Knapp, M. (2018). Sources and directions of social support and life satisfaction among solitary Chinese older adults in Hong Kong: The mediating role of sense of loneliness. Clinical Interventions in Aging, 13, 63-71. doi:10.2147/cia.s148334

Basabe, N., Páez, D., Aierdi, X., \& JiménezAristizabal, A. (2009). Salud e inmigración. Aculturación, bienestar subjetivo y calidad de vida. Bilbao: Ikuspegi y UPV/EHU.

Bastida-Gonzalez, R., Valdez-Medina, J. L., Valor-Segura, I., Fuentes, N., \& RiveraAragon, S. (2017). Marital satisfaction and marital status as a protective factor of depression and anxiety. Revista Argentina De Clinica Psicologica, 26(1), 95-102. doi:10.24205/03276716.2017.1008

Bayrami, M., Heshmati, R., \& Karami, R. (2011). Anxiety: Trait/sate, sensation seeking and marital satisfaction in married women. In D. E. Ongen, C. Hursen, M. Halat \& H. Boz (Eds.), 2nd World Conference on Psychology, Counselling and Guidance-2011 (Vol. 30).

Berkman, L. F. (1995). The role of social relations in health promotion. Psychosomatic Medicine, 57(3), 245-254.

doi:10.1097/00006842-199505000-00006

Bermúdez, E. (2004). Imágenes de salud y enfermedad de las mujeres colombianas inmigrantes en España. Revista Gerencia y Políticas de Salud, 3(7), 78-100.

Bulgan, G., \& Çiftçi, A. (2017). Psychological adaptation, marital satisfaction, and academic self-efficacy of international students. Journal of International Students, 7(3), 687-702. doi: $10.5281 /$ zenodo.570028

Bürgelt, P. T., Morgan, M., \& Pernice, R. (2008). Staying or returning: Pre-migration influences on the migration process of German migrants to New Zealand. Journal of Community and Applied Social Psychology, 18(4), 282-298. doi:10.1002/casp.924

Cabrera, V. E., Guevara, I. P., \& Barrera, F. (2006). Relaciones maritales, relaciones paternas y su influencia en el ajuste psicológico de los hijos. Acta Colombiana de Psicología, 9(2), 115-126.

Calvo, R., Carr, D. C., \& Matz-Costa, C. (2017). Another paradox? The life satisfaction of older Hispanic Immigrants in the United States. Journal of Aging and Health, 29(1), 324. doi:10.1177/0898264315624901

Carr, D., Freedman, V. A., Cornman, J. C., \& Schwarz, N. (2014). Happy marriage, happy life? Marital quality and subjective well-being in later life. Journal of Marriage and Family, 76(5), 930-948. doi:10.1111/jomf.12133

Cohen, S., Underwood, L. G., \& Gottlieb, B. H. (2000). Social support measurement and intervention: A guide for health and social scientists. Oxford: Oxford University Press.

Cooper, K., Chassin, L., \& Zeiss, A. (1985). The relation of sex-role, self-concept and sex-role attitudes to the marital satisfaction and personal adjustment of dual-worker couples with preschool-children. Sex Roles, 12(1-2), 227-241. doi:10.1007/bf00288049

Diaz-Veiga, L. (1985). Evaluación del apoyo social y comportamiento afectivo en ancianos. Madrid: Universidad Autónoma de Madrid.

Díaz, J., \& Sánchez, M. P. (2001). Relevancia de los estilos de personalidad y las metas personales en la predicción de la satisfacción vital. Anales de psicología, 17(2), 151-158.

Diener, E., Diener, M., \& Diener, C. (1995). Factors predicting the subjective well-being of nations. Journal of Personality and Social Psychology, 69(5), 851-864. doi:10.1037/0022-3514.69.5.851

Diener, E., Emmons, R., Larsen, R., \& Griffin, S. (1985). The satisfaction with life scale. The Journal of Personality Assessment, 49, 71-75. doi:10.1207/s15327752jpa4901_13

Domínguez, J. M. (2006). Apoyo Social, integración y calidad de vida de la mujer inmigrante en Málaga. Unpublished Doctoral thesis. Universidad de Málaga.

Elgorriaga, E., Martínez-Taboada, C., \& Arnoso, A. (2014). La importancia del género y el 
origen cultural en los procesos migratorios y en la satisfacción con la vida de la población inmigrante. Boletín de Psicología, 112, 7-34.

Fernández-Berrocal, P., \& Extremera, N. (2009). La inteligencia emocional y el estudio de la felicidad. Revista Interuniversitaria de Formación del Profesorado, 66, 85-108.

Field, A. (2005). Discovering statistics using SPSS. London: SAGE.

Frazier, P. A., Tix, A. P., \& Barron, K. E. (2004). Testing moderator and mediator effects in counseling psychology research. Journal of Counseling Psychology, 51, 115-134. doi:10.1037/0022-0167.51.1.115

García, M. A., \& Hombrados, M. I. (2003). La participación social en los Consejos Municipales de Menores. Encuentros en Psicología Social, 1(3), 187-190. doi:10.18004/riics.2016.julio.87-102

Gómez, V., Villegas, C., Barrera, F., \& Cruz, J. E. (2007). Factores predictores de bienestar subjetivo en una muestra colombiana. Revista Latinoamericana de Psicología, 39(2), 311325.

Gregorio, C. (1998). Inmigración, identidad de género y choque cultural: El caso de las mujeres dominicanas. OFRIM Suplemento(161-174).

Guo, M. (2014). Parental status and late-life wellbeing in rural China: The benefits of having multiple children. Aging Ment Health, 18(1), 19-29. doi:10.1080/13607863.2013.799117

Hayes, A. F. (2013). Introduction to mediation, moderation, and conditional process analysis: A regression-based approach. New York: Guilford Press.

Hepworth, D., \& Larse, J. (1986). Direct social work practice: Theory and skills. Chicago, IL: The Dorsey Press.

Hiller, H. H., \& McCaig, K. S. (2007). Reassessing the role of partnered women in migration decision-making and migration outcomes. Journal of Social and Personal Relationships, 24(3), 457-472. doi:10.1177/0265407507077233

Holgado, F. P., Sánchez, A., \& Navas, L. (2011). Análisis de la estructura de la Escala de Actitudes hacia la Inmigración en una muetsra de estudiantes chilenos. Revista
Iberoamericana de Diagnóstico y Evaluación - e Avaliação Psicológica, 29(1), 97-113.

Hudson, W. W. (1992). The WALMYR assessment scales scoring manual. Tempe, AZ: WALMYR Publishing Co.

Im, H. (2010). The impact factors on mental health among marriage migrant women. Mental Health \& Social Work, 34, 189-221.

INE (2017). Cifras de Población a 1 de enero de 2017. Estadística de Migraciones 2016. Datos Provisionales Available from http://www.ine.es/prensa/cp_2017_p.pdf

Iraurgi, I., Sanz, M., \& Martínez-Pampliega, A. (2004). Funcionamiento familiar y severidad de los problemas asociados a la adicción a drogas en personas que solicitan tratamiento. Adicciones, $\quad 16(3), \quad$ 185-196. doi:10.20882/adicciones.400

Khawaja, N. G., Yang, S., \& Cockshaw, W. (2016). Taiwanese migrants in Australia: An investigation of their acculturation and wellbeing. Journal of Pacific Rim Psychology, 10. doi:10.1017/prp.2016.1

Kong, F., \& You, X. (2013). Loneliness and selfesteem as mediators between social support and life satisfaction in late adolescence. Social Indicators Research, 110(1), 271-279. doi:10.1007/s11205-011-9930-6

Lin, N., Dean, A., \& Ensel, W. M. (1986). Social support, life events and depression. Nueva York: Academic Press.

Maglio, A. L., Molina, M. F., Raimundi, M. J., González, M. A., \& Schmidt, V. (2014). El apoyo familiar en la interfaz familia-trabajo. Construcción de una escala para su evaluación. Revista Iberoamericana de Diagnóstico y Evaluación - e Avaliação Psicológica, 37(1), 187-202.

Moreno-Jiménez, M. P., \& Hidalgo, M. C. (2011). Medición y predicción de la satisfacción con la vida en inmigrantes empleados en España. Anales de Psicología, 27(1), 179-185.

Moscato, G. (2012). Familias interculturales en España: Análisis de la satisfacción vital. Portularia, $\quad 12\left(\mathrm{~N}^{\circ} \quad\right.$ Extra $), \quad 35-43$. doi:10.5218/prts.2012.0004

Moscato, G., Novara, C., Hombrados-Mendieta, I., Romano, F., \& Lavanco, G. (2014). Cultural identification, perceived discrimination and sense of community as 
predictors of life satisfaction among foreign partners of intercultural families in Italy and Spain: A transnational study. International Journal of Intercultural Relations, 40, 22-33. doi:10.1016/j.ijintrel.2013.11.007

Neto, F. (1995). Predictors of satisfaction with life among second generation migrants. Social Indicators Research, 35(1), 93-116. doi:10.1007/BF01079240

Nho, C. R., Kim, J. H., Shin, H. J., \& Heo, S. H. (2017). Meta-analysis of depression among marriage-based migrant women in South Korea. Asian Social Work and Policy Review, 11(3), 205-215. doi:doi:10.1111/aswp.12126

Noble, R. E. (2005). Depression in women. Metabolism, 54(5 Suppl. 1)(49-52).

Nolen-Hoeksema, S. (2001). Gender differences in depression. Current Directions in Psychological Science, 10, 173-176. doi:10.1111/1467-8721.00142

Observatorio Vasco de Inmigración (2018). Evolución de la población total y de origen extranjero en la CAE y territorios históricos. 1998-2018. Recuperado de: http://www.ikuspegi.eus/documentos/tablas/ca stellano/evolucion/2018/Evolucion_extranj_to tal_2018.xlsx.

Ramirez, A. (1998). Migraciones, género e Islam. Mujeres marroquíes en España. Madrid: Agencia Española de Cooperación internacional.

Rico, N. (2006). Las Mujeres latinoamericanas en la migración Internacional. Paper presented at the Segundo Foro Mundial de las Migraciones.

Rubin, M. (2013). "It wasn't my idea to come here!": Ownership of the idea to immigrate as a function of gender, age, and culture. International Journal of Intercultural Relations, 37(4), 497-501. doi: 10.1016/j.ijintrel.2013.02.001

Shevlin, M. E., Brunsden, V., \& Miles, J. N. V. (1998). Satisfaction With Life Scale: Analysis of factorial invariance, meanstructures and reliability. Personality and Individual Differences, 25, 911-916. doi: 10.1016/S01918869(98)00088-9

Taylor, S., \& Repetti, R. (1997). Health psychology: What is unhealthy environment and how does it get under skin? Annuals Reviews in Psychology, 48, 411-447. doi:10.1146/annurev.psych.48.1.411

Thomas, E. J. (1997). Marital communication and decision making. New York: Free Press.

Utrera, S. G., \& Guardiola, J. (2017). Life satisfaction of foreign people in Spain during the economic crisis. OBETS, 12(1), 61-85. doi:10.14198/OBETS2017.12.1.03 